\title{
Evaluation of Effective Vehicle Probe Information Delivery with Multiple Communication Methods
}

\author{
Tatsuya Yamada, Mayu Mitsukawa, Hideki Shimada, Kenya Sato \\ Mobility Research Center, Doshisha University, Kyoto, Japan \\ Email: ksato@mail.doshisha.ac.jp
}

Received 15 January 2015; accepted 28 March 2015; published 31 March 2015

Copyright (C) 2015 by authors and Scientific Research Publishing Inc.

This work is licensed under the Creative Commons Attribution International License (CC BY). http://creativecommons.org/licenses/by/4.0/

(c) (i) Open Access

\begin{abstract}
Vehicle probe information delivery systems can be broadly divided into the center type and center-less type. Since conventional center-type information delivery systems generate a large load on the communications infrastructure and data center, research efforts have come to be focused on the center-less type. However, existing vehicle probe information delivery systems suffer from various problems including a limited service area, low delivery efficiency, and lack of immediacy in delivery. Our objective in this study is efficient delivery of vehicle probe information as needed. We propose a delivery scheme that uses vehicle-to-vehicle communication, infrastructure-to-vehicle communication, and mobile communication as well as Geo cast. This combined use of multiple communication methods achieves efficient information delivery by changing the communication method to fit the current situation. The results of an evaluation by simulation showed that the proposed scheme could deliver information efficiently in a variety of environments.
\end{abstract}

\section{Keywords}

Vehicle Probe Information, ITS, Style, Vehicle-to-Vehicle Communication

\section{Introduction}

Research has been active in recent years on the delivery of vehicle probe information as one type of service using vehicle-to-vehicle (V2V) communication [1]. The methods used for delivering vehicle probe information can be broadly divided into the center type and center-less type. The center type uses either infrastructure-to-vehicle (I2V) communication or mobile communication: the former collects and provides vehicle probe information through roadside units while the latter does so through mobile terminals. The center-less type, meanwhile, uses 
V2V communication. Exchanging information directly between vehicles is a method that excels in immediacy and makes infrastructure such as roadside units and base stations unnecessary. Since conventional center-type information delivery systems generate a large load on the communications infrastructure and data center, research efforts have come to be focused on center-less information delivery systems. Vehicle probe information collected and provided in one of the ways described above can be used to support a Driving Safety Support System, to alleviate congestion, etc. However, existing vehicle probe information delivery systems suffer from various problems including a limited service area, low delivery efficiency, and lack of immediacy in delivery. Our objective in this study is efficient delivery of vehicle probe information as needed, where efficient delivery is defined as the provision of information with high throughput and low delay. Specifically, we propose a delivery scheme that makes use of V2V communication, I2V communication, and mobile communication. This combined use of multiple communication methods achieves efficient information delivery by changing the communication method to fit the current situation.

\section{Vehicle Probe Information Delivery Systems}

\subsection{System Configuration}

A vehicle probe information delivery system treats a vehicle itself as a single moving sensor (referred to below as the "ego vehicle"). This system collects vehicle sensor information by various communication means and consolidates and processes that information to support a Driving Safety Support System, alleviate congestion, improve the environment, etc. Examples of services using a vehicle probe information delivery system include the delivery of hazardous location information based on driving behavior and optimal route guidance using congestion information.

\subsection{Problems with Existing Delivery Systems}

Vehicle probe information delivery systems can be broadly divided into the center type and center-less type. Infrastructure-to-vehicle communication employs Dedicated Short Range Communications (DSRC) [2]. In this type of communication, inter-vehicle interference cannot easily occur, which means reliable communications. However, the effective communication distance in DSRC is relatively short making for a limited service area. Mobile communication, meanwhile, uses wide-area radio communications enabling the collection and provision of information regardless of where the vehicle may be in an unlimited service area. On the other hand, the collection and provision of information in mobile communication is all achieved via transmissions with a data center, which detracts from immediacy.

In contrast to the above, V2V communication performs transmissions directly between vehicles without the need for base stations or other infrastructure facilities, which means a method that excels in immediacy. This method, however, collects and provides information only between vehicles. As a result, a small number of vehicles can make it difficult to collect and provide information while a large number of vehicles can generate congestion in the network resulting in a drop in delivery efficiency.

\section{Proposed Scheme}

\subsection{Objective}

In this study, we propose a delivery scheme that uses V2V communication, I2V communication, or mobile communication and Geocast communication as well. This combined use of multiple communication methods can achieve efficient delivery of probe information by switching the communication method as conditions change. Specifically, the scheme switches the method to be used by a vehicle according to the number of surrounding vehicles and to whether a roadside unit is present in the vicinity. The scheme will use V2V communication or I2V communication to deliver information that demands immediacy such as that for a Driving Safety Support System and will use mobile communication for supplementary information such as meteorological data. Vehicle-to-vehicle communication, in particular, will deliver information using Geocast communication to achieve efficient dissemination of information.

The target here is the delivery of traffic information and information that can be used to provides services to vehicles. A variety of applications can be envisioned including a Driving Safety Support System and the deli- 
very of congestion reports, meteorological data, road conditions, and disaster information. With reference to materials [3] specifying delay-time requirements, we have established requirements for delay time with respect to a Driving Safety Support System that we envision to be the main application of our proposed scheme. Delaytime requirements differ depending on the support level.

\subsection{Operation of Proposed Scheme}

\subsubsection{Switching of Communication Methods}

The switching of communication methods in the proposed scheme depends on the number of vehicles surrounding the ego vehicle and the nearby presence of a roadside unit. The ego vehicle obtains the number of surrounding vehicles by using hello packets that it transmits by packet flooding limited to one hop. This enables the ego vehicle to count the number of vehicles with which a connection could be achieved and to then decide on the communication method to be used. Specifically, if the number of surrounding vehicles is equal to or greater than $\mathrm{n} 1$ and less than $\mathrm{n} 2$, the ego vehicle uses $\mathrm{V} 2 \mathrm{~V}$ communication, and if less than $\mathrm{n} 1$ or equal to or greater than $\mathrm{n} 2$, it uses mobile communication. In addition, the ego vehicle determines whether a roadside unit is nearby by using location information on the roadside unit and location information on itself. Location information on the roadside unit is obtained by referring to data recorded on a digital map within a vehicular application. The ego vehicle can then compare this data with its own location information obtained from the Global Positioning System (GPS) to obtain its distance from the roadside unit. If the roadside unit exists within a radius of Lm from the vehicle, the ego vehicle uses I2V communication.

Furthermore, in the proposed scheme, if peripheral conditions (number of surrounding vehicles and presence/ absence of a nearby roadside unit) should change, the communication method will change in real time to match current conditions. Throughput and delay time in this scheme will therefore differ compared with delivery when using only a single communication method.

The operation of the proposed scheme is shown in Figure 1. In the figure, $L$ denotes the distance between the vehicle and roadside unit and $\mathrm{n} 1$ and $\mathrm{n} 2$ denote the number of surrounding vehicles. The layered structure of the proposed scheme is as follows. First, on the application layer, the proposed scheme runs applications for V2V communication. These applications generate transmit data based on data obtained from vehicular devices, manage that transmit data, and determine the transmit area. Next, on the network layer, the proposed scheme transmits that data using Geocast or unicast communication. Finally, on the physical layer, the proposed scheme uses a network interface according to the communication method being used.

\subsubsection{Use of V2V Communication}

Delivery by V2V communication is used in an environment in which the amount of vehicular traffic is high so that a sufficient amount of information can be exchanged among vehicles. Specifically, V2V communication is used if the number of surrounding vehicles is equal to or greater than $\mathrm{n} 1$ and less than $\mathrm{n} 2$. In this case, information delivery to another vehicle is performed through Geocast [4] communication. A Geocast refers to a form of communication that uses location information instead of node IDs when transmitting data in an ad hoc network. In general, important factors in establishing communications between two nodes in an ad hoc network are the destination node ID and transmission path. In contrast, a key feature of Geocast communication is that there is no need to specify node IDs as is necessary in ordinary multicast communications, which means that there is no

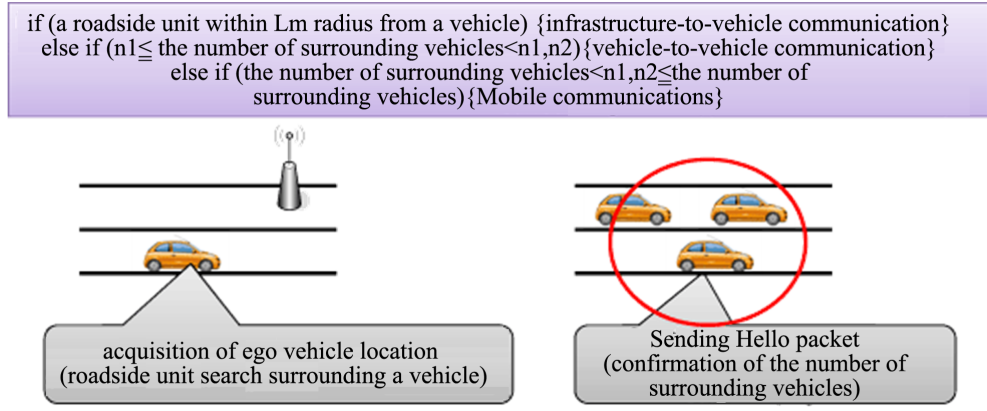

Figure 1. Proposed method. 
need to learn the IDs of the destination nodes. In multicast communication, the packet header holds the destination node ID, while in Geocast communication, the packet header holds the ego vehicle's location information obtained using GPS together with information on the destination area. In this way, V2V communication transmits data to nodes in the destination area with individual vehicles performing relay transmission up to the destination area.

For relay transmission, the proposed scheme sets the destination area (Geocast area) and the relay area (forwarding area) from the location information specifying the destination. The setting of each of these areas is performed by vehicular applications. The Geocast area is set as a fixed area based on destination location coordinates. The forwarding area is set within the range of traffic lanes in both directions based on current location information of the information source and on road information. Here, road information is obtained from a map system that can detect the ego vehicle's position on the road from current location information. The forwarding area is set so as to encompass the information source and the Geocast area. Furthermore, in transmission by the proposed scheme, the following types of data are added to the transmit data: destination location information (destination coordinates representing a geographical location), ego-vehicle location information (information obtained from GPS), information set by vehicular applications (information indicating the range of the Geocast area and forwarding area), and time to live (TTL) information (to limit the range of flooding).

In relay transmission by the proposed scheme, the information source performs data flooding. A nearby vehicle that receives that data observes that the TTL of that packet is set to 1 , which indicates that no re-flooding is to be performed. Next, this vehicle determines whether it belongs to the forwarding area based on location information obtained from GPS and forwarding-area information in the packet. If it does not belong, the data is destroyed. However, if the vehicle does belong to the forwarding area, it uses information on the destination area to transmit the data to the vehicle closest to the destination area among the vehicles situated within its transmission range. The data can therefore be relayed to the Geocast area by repeating this transmission process. Furthermore, if the ego vehicle should determine that it belongs to the Geocast area, it performs flooding to deliver the data to the vehicles in the destination area. When an ego vehicle sets out to transmit data to the vehicle closest to the destination area among vehicles within its transmission range, it limits candidate vehicles to those within the forwarding area and determines the location of nearby vehicles by exchanging hello packets with them. In this way, the ego vehicle determines its positional relationship with nearby vehicles enabling it to decide which of those vehicles will be its transmission destination.

Relaying data using only vehicles within the forwarding area in this way reduces the communications load on the network. In addition, including a vehicle's orientation information in vehicle probe information can further limit the delivery area. For example, orientation information can be used to propagate information on a sudden stop only to vehicles behind the ego vehicle thereby keeping the amount of communication needed to a bare minimum.

\subsubsection{Use of I2V Communication}

Delivery by I2V communication is used in an environment having a high vehicle density and including, for example, a traffic intersection at which a roadside unit is installed. In particular, I2V communication is used if a roadside unit exists within a distance of Lm from the vehicle. Location information on the roadside unit is obtained by referring to data recorded on a digital map within a vehicular application. An ego vehicle obtains its own location information and compares it with that of the roadside unit to decide whether to use I2V communication.

\subsubsection{Use of Mobile Communication}

Delivery by mobile communication is used in an environment in which the amount of vehicular traffic is low making the passing of information to another vehicle difficult, or in an environment in which vehicle density is so high so that V2V communication would generate congestion in the network. Specifically, mobile communication is used if the number of surrounding vehicles is less than $\mathrm{n} 1$ or equal to or greater than $\mathrm{n} 2$. In general, mobile communication is used to deliver information that does not demand immediacy compared to other types of information. Mobile communication uses the $3 \mathrm{G}$ network and can therefore deliver information within a service area that is essentially unlimited. It also enables the provision of information using pull-type communication depending on the needs of the driver. 


\section{Performance Evaluation by Simulation}

\subsection{Simulator}

In this study, we used the Qualnet [5] simulator to evaluate the performance of the proposed scheme. The parameters used in this simulation are listed in Table 1. For packet size, we adopted the value generally used in papers related to V2V communication [6]-[8]. Furthermore, with the aim of replicating communications in an actual environment, we used TWO-RAY as the spatial model since it takes ground-reflected waves into account [6]. Additionally, we decided on antenna height and radio output range by referring to transmission antennas mounted on ordinary vehicles [7]. Finally, for the MAC layer and packet size, we adopted the system and value generally used in V2V communication and having a proven track record in research and development [6]-[8].

\subsection{Evaluation Models}

To compare the proposed scheme and existing schemes in terms of delivery efficiency and delivery immediacy, we measured throughput (amount of data transmitted per second) and delay time (time taken for data transmitted from the information source to arrive at its destination) for various evaluation models as described below.

\subsubsection{Evaluation Model 1}

For evaluation model 1, we simulated delivery by various communication systems that can be used for V2V communication with the aim of demonstrating the usefulness of Geocast as used in V2V communication by the proposed scheme. To test throughput and delay time under conditions in which V2V communication can generally be used, we assumed an environment in which groups of vehicles pass each other within an area having an intersection. Given this environment and referring to Advanced Safety Vehicle (ASV) study materials [9] of the Japan Automobile Manufacturers Association (JAMA), we set driving speed to $45 \mathrm{~km} / \mathrm{h}$, inter-vehicle distance to $20 \mathrm{~m}$, and number of vehicles to $10-20$. We assumed that transmit data would consist of approaching-vehicle information and set its size to $2 \mathrm{MB}$ including graphics and text data, which is basic to vehicle probe information. The specific targets of comparison that we used for this evaluation were communication systems commonly used for V2V communication, namely, flooding (with TTL limitations set so that no retransmission occurs when the same data is received multiple times), Optimize Link State Routing (OLSR), Ad hoc on-Demand Distance Vector (AODV), and Geocast. Evaluation model 1 is outlined in Figure 2.

\subsubsection{Evaluation Model 2}

For evaluation model 2, we simulated delivery when changing parameters $(\mathrm{n} 1, \mathrm{n} 2)$ in the proposed scheme with the aim of deriving the optimal values for those parameters. Here, to assess throughput and downlink for various combinations of $\mathrm{n} 1$ and $\mathrm{n} 2$ in the delivery of vehicle probe information in an actual environment, we referred to ITS Communication Simulation Evaluation Scenarios [9] and performed the simulation for an environment in which some vehicles were traveling in the east-west direction and other vehicles were stationary in the north-south direction within a $2.5 \times 3.5 \mathrm{~km}$ area. Furthermore, referring again to ASV study materials of JAMA, we set driving speed to $60 \mathrm{~km} / \mathrm{h}$, inter-vehicle distance to $45 \mathrm{~m}$, and number of vehicles acting as information sources to 10 - 40. Transmit data consisted of 3 MB of sensor data (graphics, etc.). In the simulation, we measured average throughput and average delay time for 10 - 40 vehicles acting as information sources. We first varied $\mathrm{n} 2$ while setting $\mathrm{n} 1$ constant to determine an optimal value for $\mathrm{n} 2$, and after determining $\mathrm{n} 2$, we varied $\mathrm{n} 1$ to determine its optimal value. Specifically, for $\mathrm{n} 2$, we adopted the value that recorded highest throughput and lowest delay time. Evaluation model 2 is outlined in Figure 3.

Table 1. Simulation parameters.

\begin{tabular}{cc}
\hline Simulator & Qualnet ver. 5.0.1 \\
\hline Spatial model & TWO-RAY: consideration of ground-reflected waves \\
Antenna height & $1.5 \mathrm{~m}$ \\
MAC layer & IEEE802.11b \\
Packet size & 512 byte \\
Radio output range (effective radius) & V2V communication: $50 \mathrm{~m}$ \\
\hline
\end{tabular}




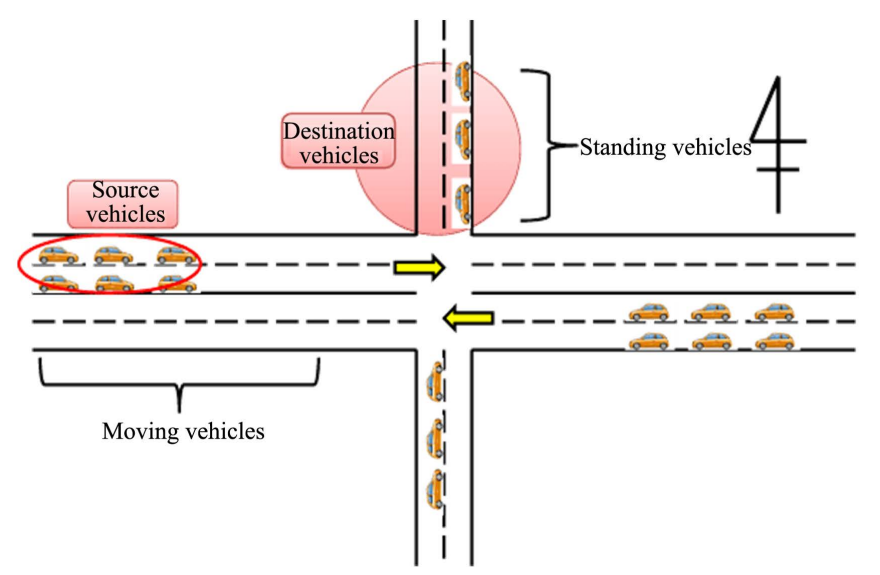

Figure 2. Outline of evaluation model 1.

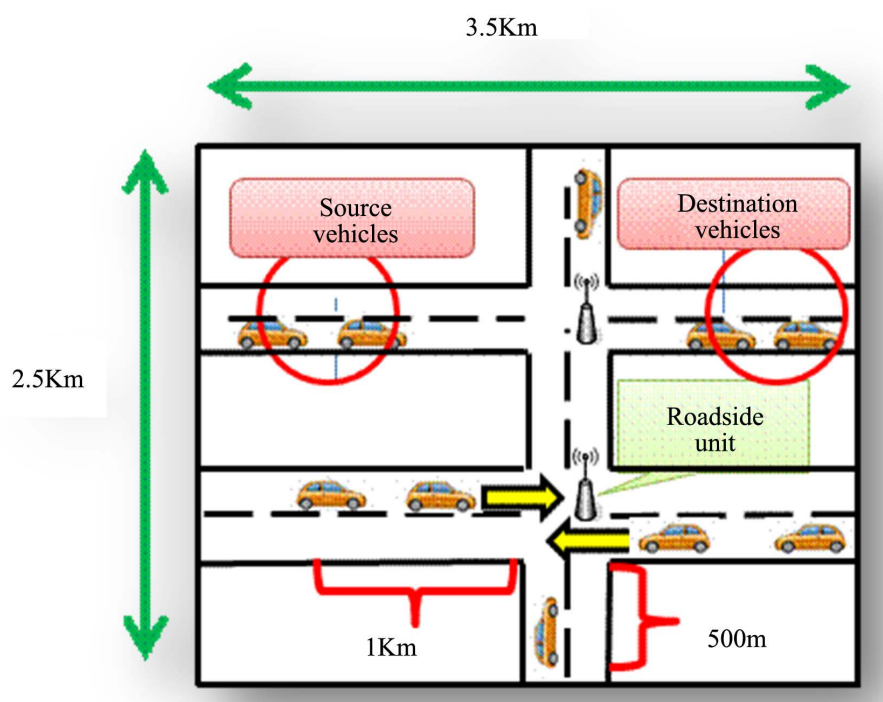

Figure 3. Outline of evaluation model 2.

\subsubsection{Evaluation Model 3}

We used evaluation model 3 to assess throughput and delay time for various techniques assuming the delivery of vehicle probe information in an actual environment. Specifically, we performed a simulation for an environment in which vehicles were traveling in the east-west direction within a $2.5 \times 3.5 \mathrm{~km}$ area. The simulation model that we used for this evaluation was the same as evaluation model 2. Referring again to ASV study materials, we set driving speed to $60 \mathrm{~km} / \mathrm{h}$, inter-vehicle distance to $45 \mathrm{~m}$, and number of vehicles acting as information sources to 10 - 40. Transmit data consisted of $3 \mathrm{MB}$ of sensor data (graphics, etc.). The communication methods that we compared in this case were V2V communication (AODV), I2V communication (amplitude-shift keying (ASK)), mobile communications ( $3 \mathrm{G}$ ), and the proposed scheme. For each of these communication methods, we adopted a communication system that is typically used for that method [10].

\subsection{Simulation Results}

\subsubsection{Evaluation Model 1}

For evaluation model 1, we measured throughput and delay time for various communication systems used in V2V communication. It was found that delivery using Geocast achieved high throughput and low delay by transmitting information only within a specific range. Simulation results for evaluation model 1 are shown in Figure 4 and Figure 5. 


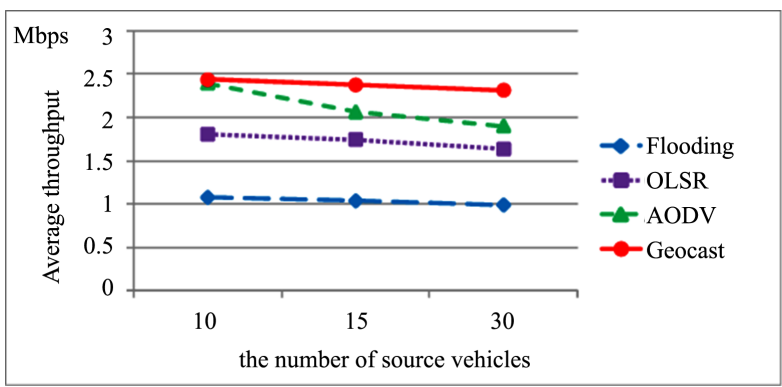

Figure 4. Results for evaluation model 1 (throughput).

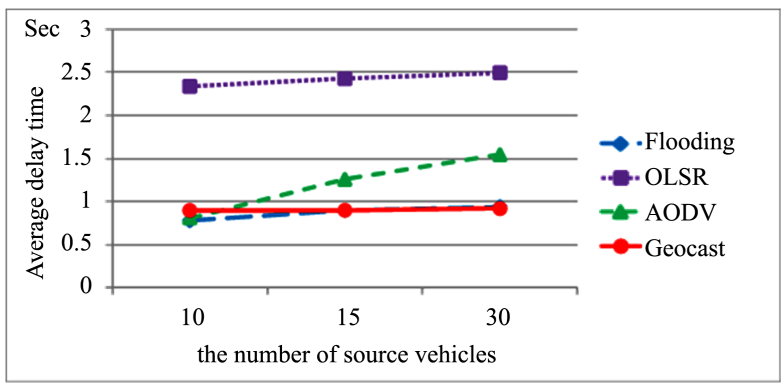

Figure 5. Results for evaluation model 1 (delay time).

\subsubsection{Evaluation Model 2}

For evaluation model 1, we measured throughput and delay time while varying parameters (n1, n2) in the proposed scheme. The results showed that high-throughput and low-delay communications could be achieved for $\mathrm{n} 1=2$ and $\mathrm{n} 2=6$, so these values were taken to be optimal for those parameters. Simulation results for evaluation model 2 are shown in Figure 6 and Figure 7. Specifically, for n2, we varied its value in the manner of 2, 3, $4,5, \ldots$ and adopted the value for which the highest throughput and lowest delay time were measured.

\subsubsection{Evaluation Model 3}

For evaluation model 3, we measured throughput and delay time for V2V communication (AODV), I2V communication (ASK), mobile communications (3G), and the proposed scheme. Results showed that the proposed scheme maintained high throughput regardless of the number of source vehicles since it was able to perform information delivery by changing the communication method as needed. The parameters used in the simulation of the proposed scheme were $\mathrm{L}=50, \mathrm{n} 1=2$, and $\mathrm{n} 2=6$. Simulation results for evaluation model 3 are shown in Figure 8 and Figure 9.

\section{Discussion}

\subsection{Evaluation Model 1}

Geocast communication reduces wasteful transmission by transmitting information only to those vehicles needing it in a limited area. This reduces the load on the network resulting in high throughput. Flooding, on the other hand, transmits data to all vehicles, which increases the load on the network and causes many packets to drop thereby reducing throughput. In OLSR, meanwhile, vehicles are always moving in an east-west direction within the communication area resulting in significant changes in the routing table. As a result, transmissions increase to update the routing table and throughput drops. AODV, in contrast, is a scheme that does not continuously maintain a routing table, so a route must be created for each destination. This means that transmissions involved in route control increase as the number of vehicles increase, which causes throughput to drop. Both Geocast and flooding require a small amount of control when transmitting data to multiple vehicles, and as a result, delay time is small. In OLSR, the routing table changes greatly when vehicles moving toward each other pass each other. Transmissions are therefore needed at this time to update the routing table, which causes delay time to increase. In AODV, the time involved in creating routes depends on the number of vehicles, so time is needed to 


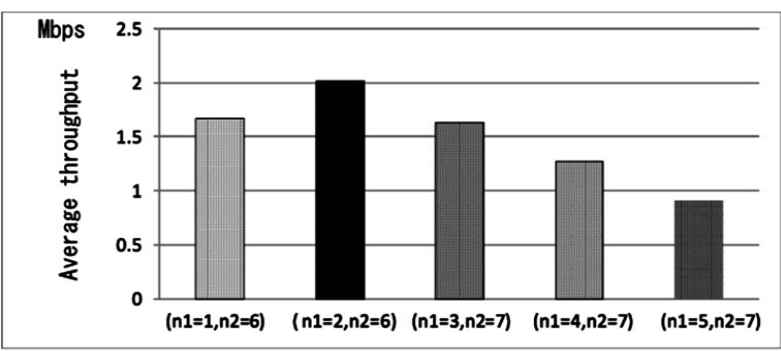

Figure 6. Results for evaluation model 2 (throughput: n2 is optimal number).

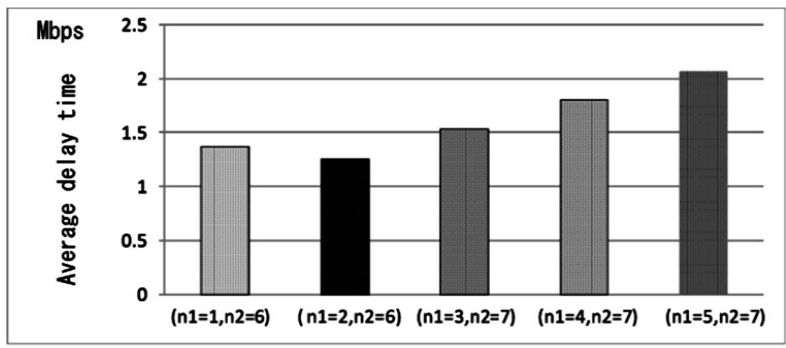

Figure 7. Results for evaluation model 2 (delay time: n2 is optimal number).

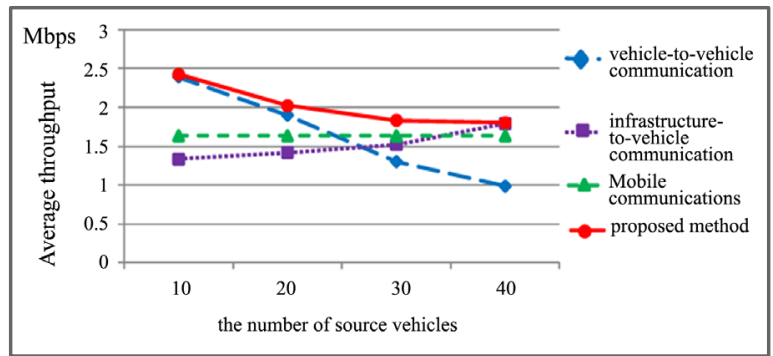

Figure 8. Results for evaluation model 3 (throughput).

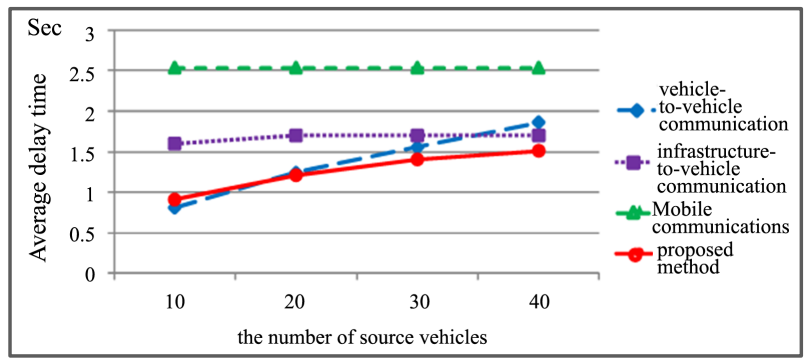

Figure 9. Results for evaluation model 3 (delay time).

establish routes in an environment with a high density of vehicles thereby increasing delay time. Based on the above discussion, delivery by Geocast is superior in terms of throughput and delay time.

\subsection{Evaluation Model 2}

The results obtained from simulations while varying $n 1$ and $n 2$ show that throughput would be low and delay time would be long if the value of $n 1$ is high or the value of $n 2$ is low since mobile communication would be used in such cases even if other vehicles are near the ego vehicle. Furthermore, for $\mathrm{n} 2$ set above a certain level (n2 $=6,7)$, it was found that throughput would be low and delay time would be long owing to network congestion 
since V2V communication would be used regardless of whether other vehicles are concentrated about the ego vehicle. Accordingly, optimal conditions for switching communication methods are the presence of other vehicles at a number that does not generate congestion in the network. Based on the results of these simulations, we concluded that these optimal conditions occur for $n 1=2$ and $n 2=6$.

\subsection{Evaluation Model 3}

In V2V communication, communication load on the network increases as the number of vehicles that act as information sources increase thereby degrading throughput. In I2V communication, an increase in the number of vehicles means that more vehicles will come to lie within the range of communication with the roadside unit, which improves average throughput. Mobile communication, meanwhile, maintains constant throughput since it uses $3 \mathrm{G}$ circuits. The proposed scheme can maintain high throughput by switching among these communication methods to deliver information. In addition, V2V communication excels in immediacy since it enables vehicles to exchange data directly among themselves without having to use a data center. Here, however, delay time increases as the number of vehicles that act as information sources increase. Meanwhile, in I2V communication, delay time increases if vehicles lie outside the range of communication with the roadside unit since regular transmissions cannot be performed. In mobile communication, delay time is long since transmissions must pass through a data center. The proposed scheme uses V2V communication as its main communication method, so average delay time is short. In addition, these simulations showed that high throughput and low delay could be achieved for parameters set to $\mathrm{L}=50, \mathrm{n} 1=2$, and $\mathrm{n} 2=6$. In short, we have shown that the proposed scheme is superior in terms of throughput and delay time compared with existing delivery schemes.

\section{Related Research}

Takahata et al. proposed a vehicle probe information delivery system using I2V communication [11]. They developed, in particular, a center-type probe information delivery system using DSRC. This system, however, can only collect and provide information in areas within the vicinity of roadside units, so it is not oriented to generating local information. Our proposed scheme, in contrast, can provide services regardless of the environment. Ito et al. proposed a vehicle probe information delivery system using V2V communication [12]. In this system, information is exchanged only between vehicles, and as a result, service quality is somewhat affected by the number of vehicles present. Our proposed scheme, meanwhile, uses multiple communication methods and is consequently unaffected by the number of vehicles, so it excels in terms of service provision.

Ushitani et al. also proposed a vehicle probe information delivery system using multiple communication methods [13]. It can collect vehicle probe information over a wide area through a communication network that combines V2V and I2V communication methods. This system looks to be applicable to traffic accident prevention, hazard avoidance, and congestion alleviation. However, the basis for communication in this system is the vehicle, and as a result, communications cannot be established in an environment having only a few vehicles. That is to say, the system assumes an area with a high concentration of vehicles even in an applicable environment-using it in an area with only a scattering of vehicles is difficult. Our proposed scheme, though, can use mobile communication even in an area with a scarcity of vehicles, so it excels in the ability to collect and provide vehicle probe information.

Finally, Komiya et al. developed an evaluation platform using a center-less type of information delivery system [14]. Their research showed that center-less type of probe information delivery is superior in terms of a $\mathrm{CO}_{2}$ reduction effect. We can therefore infer that our proposed scheme is a feasible application.

\section{Conclusions}

In this study, we proposed a delivery scheme that uses vehicle-to-vehicle communication, road-to-vehicle communication, or mobile communication to enable efficient delivery of large volumes of information without limiting the service area. Combining multiple communication methods in this way enables the communication method to be changed depending on current conditions so that information can be delivered in the most efficient way.

In simulations that we performed to evaluate the proposed scheme, we measured throughput and delay time for both the proposed scheme and existing schemes in envisioned environments. Simulation results revealed that 
switching communication methods by the proposed scheme maintained high throughput and low delay and achieved efficient delivery of data. These results demonstrate that the proposed scheme is superior to existing schemes in terms of delivery efficiency and delivery immediacy.

\section{Acknowledgements}

This research was supported partly by a Grant-in-Aid for Scientific Research (24300030) and the Strategic Information and Communications R\&D Promotion Programme (12180615) by the Ministry of Internal Affairs and Communications, Japan.

\section{References}

[1] Japan Automobile Research Institute. http://www.jari.or.jp/

[2] Kenney, J.B. (2011) Dedicated Short-Range Communications (DSRC) Standards in the United States. Proceedings of the IEEE, 99, 1162-1182. http://dx.doi.org/10.1109/JPROC.2011.2132790

[3] ETSI (2013) Intelligent Transport Systems V2X Application Road Hazard Signalling Application Requirements Specification. http://www.etsi.org/deliver/etsi_ts/101500_101599/10153901/01.01.01_60/ts_10153901v010101p.pdf

[4] Navas, J.C. and Imielinski, T. (1997) GeoCast-Geographic Addressing and Routing. Proceedings of International Conference on Mobile Computing and Networking (MobiCom), Budapest, 26-30 September 1997, 66-76.

[5] Scalable Network Technologies. http://www.scalable-networks.com/

[6] Haerri, J., Filali, F. and Bonnet, C. (2006) Performance Comparison of AODV and OLSR in VANETs Urban Environments under Realistic Mobility Patterns. Proceedings of the 5th IFIP Med-Hoc-Net'06, Lipari, June 2006, 32663277.

[7] Harri, J., Filali, F. and Bonnet, C. (2006) Mobility Models for Vehicular Ad Hoc Networks: A Survey and Taxonomy, Technical Report RR-06-168, Institute Eurecom, Sophia Antipolis.

[8] Zhao, J., Zhang, Y. and Cao, G.H. (2007) Data Pouring and Buffering on the Road: A New Data Dissemination Paradigm for Vehicular Ad Hoc Networks. IEEE Transactions on Vehicular Technology, 56, 3266-3277. http://dx.doi.org/10.1109/TVT.2007.906412

[9] Japan Automobile Research Institute, Committee Promoting the Use of ITS Simulators, ITS Communication Simulation Evaluation Scenarios, 2012. (In Japanese) http://www.jari.or.jp/Portals/0/resource/pdf/H23_simyu/\%EF\%BC\%88Ver1.2\%EF\%BC\%8920131010.pdf

[10] Japan Automobile Research Institute, ITS Research Division, Report on Feasibility Study on Development of CenterLess Probe Information System, 2007.

[11] Takahata, K., Noda, T. and Kamba, K. (2009) Information Provision System Using DSRC (Spot Communications). http://www.hrr.mlit.go.jp/library/happyoukai/h22/ino_2/209.pdf

[12] Ito, H. (2011) Developing Decentralized Probe Information Systems Utilizing 700MHz Band for Mobile Communication and Advertizing Activity on Its Achievements. JARI Research Journal, 33, 55-58.

[13] Ushitani, Y., Imao, M., Higashino, T., Tsukamoto, K. and Komaki, S. (2006) Improvement Effect in Link Blocking Rate for Joint Inter-Vehicle and Road-to-Vehicle Communication System with Large-Sized Vehicle Operation. B-Abstracts of IEICE TRANSACTIONS on Communications, J89-B, 909-919.

[14] Komiya, T., Horiguchi, R. and Koide, K. (2011) Estimation of $\mathrm{CO}_{2}$ Reduction with the "Center-Less" Probe System. Proceedings of the 10th ITS Symposium. (In Japanese) http://www.transport.iis.u-tokyo.ac.jp/publication/2011-32.pdf 